\title{
Title: Generating mutual recovery in creative spaces
}

\section{Author Details:}

Victoria Stewart

School of Human Services and Social Work, Griffith University, Brisbane, Australia

Menzies Health Institute Queensland, Griffith University, Brisbane, Australia

Helena Roennfeldt

School of Human Services and Social Work, Griffith University, Brisbane, Australia

Maddy Slattery

School of Human Services and Social Work, Griffith University, Brisbane, Australia

Menzies Health Institute Queensland, Griffith University, Brisbane, Australia

Amanda J Wheeler

School of Human Services and Social Work, Griffith University, Brisbane, Australia

Menzies Health Institute Queensland, Griffith University, Brisbane, Australia

Faculty of Medical and Health Sciences, University of Auckland, Auckland, New Zealand

Corresponding author: Victoria Stewart

v.stewart@griffith.edu.au

Acknowledgments (if applicable): The research team wish to thank the Brisbane South PHN (formerly Greater Brisbane Metro South Medicare Local) for funding this research. The views and opinions expressed in this paper are those of the authors and do not necessarily reflect those of the PHN. We also acknowledge the artists, peer mentors and research staff who participated in parts of the research journey, particularly Rani Scott and Rachael Krinks. Finally, but not least, we would like to thank our research participants without whom this project would not have been possible.

\section{Biographical Details (if applicable):}

Victoria Stewart MPhil, BOccThy GCHighEd, Lecturer, School of Human Services and Social Work and Menzies Health Institute Queensland, Griffith University, Brisbane, Australia. ORCID iD: 0000-0003-2892-2288

Helena Roennfeldt BPscySc MMHPrac MSuicide MForMH, MSW(Q), Adjunct Research Fellow, Menzies Health Institute Queensland, Griffith University, Brisbane, Australia

Maddy Slattery PhD, Senior Lecturer, School of Human Services and Social Work Menzies Health Institute Queensland, Griffith University, Brisbane, Australia

Amanda J Wheeler BPharm BSc PhD PGDipPsychPharm PGCertPubHealth, Professor of Mental Health, Griffith Health Institute, Griffith University, Brisbane, Australia and Clinical Associate Professor, Faculty of Medical and Health Sciences, University of Auckland, Auckland, New Zealand.

\section{Keywords:}

Mental health, recovery, creative arts, self-stigma, peer support

Article Classification:

Primary research paper 


\section{Abstract}

Purpose - Participation in creative activities have been linked with increased personal agency. This article addresses critical considerations in the development of community-based creative workshops for people experiencing severe and persistent mental illness and explores participant experiences of these workshops.

Design/methodology/approach - The workshops aimed to build the skills and capacities of participants and provide alternative ways to communicate identity and recovery stories. They were designed to provide a range of creative opportunities for participants (visual arts, writing, dance and music) and were facilitated by practising artists. Eleven participants attended ten creative workshops over three months. On completion, a focus group was conducted to gather participant views, experiences and outcomes of the workshops.

Findings - The creative workshops supported the recovery of participants. The inclusion of peer mentors in the workshops was an important in facilitating connectedness.

Originality/value - This study advances the evidence that creative arts can support the psychological and social aspects of participants' recovery journeys. Creative activities can promote positive self-identity and reduce self-stigma for people experiencing mental illness. It highlights the importance of using evidence in the design of creative workshops and supports the use of peer mentoring in group creative processes.

Keywords: Mental health, recovery, creative arts, self-stigma, peer support 


\section{Introduction}

This research explores the development of a series of creative workshops (Your Story Matters) which were aimed at assisting people experiencing severe and persistent mental illness to explore their story of recovery journeys. The design of the workshops will be discussed, including the purpose and staffing, followed by the key findings from a qualitative study which aimed to understand how the participants experienced the workshops.

\section{Creative arts in mental health}

The creative impulse is fundamental to human experience (Elliott, 2005). Engaging in creative activities has been found to stimulate imagination and reflection, contribute to the construction of identity and enable self-expression, inspire change and growth, engender a sense of belonging and promote healing (APPGAHW, 2017). Creative activities such as photography, painting, dance, and storytelling have been found to enhance a sense of personal identity by enabling people to become aware of their latent abilities and develop coping strategies to manage symptoms (Spandler et al., 2007). Arts participation has also been associated with rediscovering and rebuilding personal identity beyond that of someone with a mental illness (Clift, 2012).

Creativity may instil a greater sense of wellbeing and control over our actions, and for people experiencing mental illness, this may translate to increased personal agency and hope (Sayers and Stickley, 2018). Arts-based methods have been utilised to access knowledge which is not easily expressed in words or to describe difficult experiences (Stuckey and Nobel, 2010). Creative arts enable aspects of health experiences to be heard, which might otherwise be silenced (Tarr et al., 2017).

\section{Creative arts and recovery}


Generating mutual recovery in creative spaces

The concept of recovery within mental health is conceptually different from a medical model, shifting from a focus on pathology and symptoms to one which emphasises rebuilding a worthwhile life, irrespective of whether or not a person continues to experience symptoms of mental illness (Tew et al., 2011). Recovery sees people beyond their illness and emphasises abilities, possibilities, interests and dreams; reclaiming valued social roles and relationships that give life value and meaning (Repper and Perkins, 2009).

Personal stories can be a useful way for individuals, treating professionals and others to understand the illness experience, the service response, and to recognise the unique situation of each person (Kirkpatrick, 2008). Elliott (2005) considers storytelling to be fundamental to the human experience: life yields stories, stories give meaning to life, and people are embedded in stories by which they shape and are shaped by their world. Stories we tell about who we are have the ability to influence the construction of identity (McAdams, 2006). Baldwin (2005) identifies that severe mental illness can compromise a person's ability to construct a coherent life story due to their ability, and opportunity, to author their own life story. It has been suggested that mental illness threatens identity and sense of self when one's personal story is displaced by dominant illness narratives focussing on deficit and dysfunction (Frank, 1995).

Integral to recovery is providing people with opportunities to develop a renewed sense of hope, agency and meaning by redefining themselves beyond an illness identity and consequent stigma. The process of reframing or restorying can provide a sense of possibility for individuals and provides an opportunity to recover the voice that illness and treatment silences, articulate trauma and loss, demystify the illness experience, refocus on the positives of the experience, consolidate gains and construct new maps for the recovery journey (Kerr et al., 2013). The use of arts in mental health can offer a holistic, collaborative and person- 
centred approach, promoting a recovery-oriented focus on a person's emotional, social and spiritual needs alongside their clinical goals (Van Lith et al., 2013).

This paper presents a creative workshop process that was implemented as part of a service evaluation project. The Your Story Matters (YSM) creative workshops were aimed at providing participants with an opportunity to explore their identity and recovery experiences within a supportive environment. The workshops utilised music, art, dance and creative writing. Through participating in the YSM workshops, it was anticipated that participants would be better prepared and empowered to share their stories of recovery with others. Rather than focus on the artistic outputs of the YSM workshops, this paper reviews the literature which informed the development of the creative workshops, describes the design of the workshops and their unfolding in practice as well as the workshop processes. A qualitative approach was utilised to improve understanding of the experiences from the participant perspective. Ethics approval was obtained from the University Human Research Ethics Committee (HSV/13/15/HREC).

\section{Method}

Creative workshop design

Purpose: The purpose of the YSM workshop was to allow participants to build skills and capacities to share stories about their recovery journey. The workshops sought to explore alternative ways to communicate identity and recovery journeys which did not rely on language or medically focussed ways of describing mental illness.

Specifically, the workshops aimed to: 
- provide a safe space for participants to explore their experiences of recovery using creative processes; and

- establish an environment which encouraged sharing, nurturing, mentoring, encouragement and support.

Group collaboration: A group format was chosen for the workshops as social inclusion is an important element of both recovery and community-based arts projects (Lawson et al., 2014). Research suggests that engagement in art-based groups can enhance the development of social skills and assist in building relationships; forming social identity through a sense of belonging to a group (Van Lith, 2015). Finally, participation in arts-based groups enhances social inclusion through the development of a sense of social wellbeing and acceptance; overcoming loneliness and social exclusion (Van Lith et al., 2013).

Peer mentors: Peers are individuals who have had their own experiences of mental illness and are able to draw on these experiences to support others (MacNeil and Mead, 2005). A defining feature of peer work is the concept of mutuality and sharing experiences within the journey towards recovery (Mead et al., 2001). The use of peer mentors builds on the use of a group format, with the inclusion of peers found to increase group identity, acceptance and social inclusion (Argyle and Winship, 2015).

The use of peer mentors in the YSM workshops was also chosen to provide education and support to participants regarding how, when and with whom they shared their story. Possible vulnerability, and the potential for trauma associated with storytelling, has been noted in the literature for people with mental illness (Happell et al., 2015). Preparation and support have been identified as important in ensuring individuals have a clear understanding of what they are hoping to achieve by sharing their story, thus enabling them to remain in control of the content they share (Happell and Bennetts, 2016). The YSM peer mentors were 
able to discuss with participants their own experiences of sharing stories of illness and/or recovery, and identify positive outcomes as well as issues and episodes of vulnerability.

Creative mediums: Creative art therapies can include a range of artistic expressions such as drama, craft, dance or music. Stuckey and Nobel (2010) reviewed arts-based research and identified four primary therapies which are used to promote health: music, visual arts, movement-based creative expression and expressive writing. Positive health outcomes have been reported for all four mediums, so a decision was made to include these mediums in the YSM workshops.

\section{Process}

Recruitment: All consumers accessing the Partners in Recovery service (recovery-oriented care coordination service for people experiencing severe and persistent mental illness) were eligible to participate in the YSM workshops. Recruitment flyers and information brochures/emails were circulated providing information about the workshops and interested participants were invited to attend an information session. The information session was held prior to the YSM workshops, during which the aim and process of the workshops, along with discussion regarding the benefits and issues surrounding the telling of personal stories, were discussed.

Workshop overview: Ten creative workshops were conducted over three months. A total of eleven participants (eight female, three male) attended the workshops, with the majority attending all ten workshops. Four artists (one female, three males; each expert in use of the four creative areas of dance, writing, visual arts and music) and three peer mentors (two female, one male) were recruited to facilitate the YSM workshops. Each creative medium (art, music, dance and writing) had its own workshop with individual artists acting as 
Generating mutual recovery in creative spaces

facilitators. A further two workshops, attended by all the artists, enabled participants to work one-on-one with their preferred creative medium. Initial activities focused on being able to connect to self and personal stories (i.e., who we are and how we are known). Mentors provided support and guidance at all workshops. Catering was provided and travel subsidies were available for participants.

Two final YSM workshops were conducted to assist participants with completing their creative projects to present at the forthcoming Mental Health Week showcase event. Participants also requested that a future planning workshop be held at the completion of the project in which they brainstormed opportunities for ongoing social connection (e.g., facebook, social events), supporting one another with respect to creative endeavours (e.g., identifying ongoing arts-based activities), and identifying ways to disseminate their stories with community members (e.g., sharing their stories with university students).

Data collection: A focus group with workshop participants was facilitated after the final workshop to gather views, experiences and outcomes of participation in the creative workshops. Nine workshop participants (seven female, two male), one artist (female) and one mentor (female) attended the focus group which was two hours in duration and digitally recorded. Focus group questions were developed by the research team, reviewed by the peer mentors and aimed to elicit an understanding of the expectations and experiences of workshop participation (e.g., interactions with others, the experience of sharing stories of recovery, changes in self-esteem and self-stigma). The focus group discussion was transcribed and a general inductive approach was utilised to analyse the data. Codes were continually refined through the analysis with checks by other members of the research team to ensure trustworthiness of the analysis (Elo et al., 2014). Discrepancies in the coding were discussed within the team until agreement themes was reached. The research team identified 
three themes that described the experiences and outcomes of participation in the creative workshops: i) connection; ii) sense of self; and iii) achievement. These themes are discussed in the next section.

\section{Key Findings}

\section{Connection}

This theme emphasised the importance of sharing, acceptance, friendship and inclusiveness in the workshops. Essential elements included respecting difference, sharing laughter and connecting with others with similar experiences. For a number of participants, engaging in the creative processes and the sense of connection to others gave them something to look forward to, motivating them to attend.

A lot of stuff has happened for me in the last couple of months including homelessness but I still came here...it was so important for me to come here and be with people that I know, friendly faces.

The importance of an inclusive, open atmosphere, and interactions with others who shared similar experiences (including mentors, researchers and artists), was articulated by all participants. Acceptance was a critical element of participants' sense of connection; it referred to an individual's acceptance of self, as well as acceptance by others.

I don't usually leave the house but I come here to be with people with the same issues that I have. Knowing that I'm not crazy, that other people struggle with the same things that I do has really helped.

Peer mentors were identified as an important element of the workshops, ensuring that participants were comfortable, accepted and supported to share their experiences. 
They made me feel like I matter. I got a text message from [a peer mentor] when I missed a workshop - so many times so much in your life you feel no one misses you.

The skills of the mentors and artists were also identified as important in maintaining the sense of safety and connection for group members.

They [mentors and artists] had integrity, generosity and authenticity. They made us feel

like we were valuable human beings and what a gift to offer someone, plus the talents that they brought. They were generous with their resources and time.

In addition to the staff and group members promoting a sense of welcome and connection, participants also reported that the spaces where the workshops were held, along with the provision of catering, were important in facilitating a sense of respect and belonging.

Yes ... to be rewarded by arriving with such hospitality I think that's just such a doorway to someone's soul personally. It made me feel very special.

\section{Sense of self}

This theme focused on the participant's understandings of their own mental health challenges and their increased confidence in articulating these challenges to others. Negative past experiences which had shaped individual's sense of competence were explored and challenged through engagement and achievement in the creative activities, alongside discussions with others.

All my life I've been told that I haven't been good enough. That's been my life and I believed it. But coming here I've been shown that maybe .... hey, I'm nearly as good as everybody else around and I can do these things. 
As a result of their increased confidence, a number of participants identified that they were now more comfortable to share about their mental illness stories with others.

Recognising the fact that yes, I do have a mental illness and I can tell anybody now, I'm not ashamed. Before [the workshops] I was like I don't really want to tell people but now ... I'm at a place where I'm comfortable and I can acknowledge it.

Exploring their life stories; challenging times, as well as moments of triumph and of success, allowed participants to feel that their stories mattered. The opportunity to participate in research was highly valued; cultivating a sense of self-value, allowing them to find meaning in illness experiences and contribute to future service developments for others.

\section{Achievement}

This theme concerned the impact of the workshops on assisting participants to try new things and develop a sense of future. Facing new situations, overcoming and coping with challenges, finding inspiration, motivation and the acquisition of new skills were identified. In addition to the artistic and creative skills, participants emphasised the importance of learning a range of new life skills such as anxiety management.

I found coming to the different workshops I experienced panic attacks at most of them. I felt that the mentors were very attuned and aware ... and they helped me to tame the symptoms.

Participants reported a renewed sense of motivation to try new things and achieve their goals; an "idea of a future". Enrolment in courses, engagement in community groups, plans to explore employment in paid peer roles and sharing their stories with others were identified by participants as goals to work towards after completing the workshops. 
Participants articulated a disappointment in the end of the workshops and expressed a desire to explore other community creative groups and continue to provide ongoing peer support to each other.

\section{Discussion}

There was universal agreement amongst participants that the creative workshops had been beneficial and had contributed to positive outcomes; particularly connectedness and enhanced self-identity.

Connection was of central importance, highlighting the positive impact of support from others who had lived experiences of mental illness. A sense of community was developed and participants described feeling accepted and that they 'mattered' (Pernice et al., 2017). The inclusion of peer mentors was an important strategy in developing a group sense of safety and mutuality, developing an atmosphere of support and acceptance, inspiring hope and empowering others (Walsh et al., 2018).

Participants also identified the importance of having input from 'real' (i.e., practising) artists, access to quality materials and the opportunity to exhibit art works to the development of new identities and increased confidence. Stories of recovery were discovered and re-storied in and through these social interactions, as was the development of a more positive self-identity. This new confidence in understanding and sharing individual stories seemed to have a ripple effect into other areas of participants' lives, increasing community access and a desire to contribute to future service delivery in areas of advocacy and peer roles.

Participants identified that the workshops did not focus on illness and allowed control over individual participation and sharing of experiences. Participants described developing a more hopeful identity; identifying meaning and celebration in their lives and experiencing 
less shame regarding their mental illness. In line with previous research (Van Lith et al., 2011), the YSM workshops contributed strongly to the psychological and social aspects of participants' recovery journeys. This supports the idea that creative interventions can increase empowerment, reduce self-stigma and support social inclusion for people experiencing severe and persistent mental illness.

Participants reported feelings of grief and disconnection when the workshops ended, requesting a follow up meeting and brainstorming ways in which the group could continue to provide support to each other and participate in creative activities. Few potential options within the local community were identified and the group were unsuccessful in obtaining further grants to continue the creative workshops. There is limited documentation of the negative effects of arts projects, but the research team felt that the time-limited nature of the workshops and the withdrawal of peer mentor and artist support was possibly a negative consequence of this research. It is recommended that future creative arts projects consider the development of sustainable support to ensure participants are not negatively impacted in this way.

As this study involved a small number of participants, the limitations of this and the generalisability of results are acknowledged. Participants were able to self-select into the study and it is assumed that this was done by those with an interest in the creative arts.

\section{Conclusion}

The YSM workshops in this study promoted connectedness, hope, identity, meaning and empowerment among participants; these are all identified elements within recovery frameworks (Stickley et al., 2018). Whilst social inclusion agendas currently promote the integration of health activities into community settings, it is clear that participants in this 
study valued interactions with others with lived experiences of mental illness. Although lived experience was an important element of the YSM workshops, feedback from participants indicated that the skills and confidence they gained from the workshops would be useful for future participation in non-mental health specific community arts activities. The workshops could be seen as a bridge to accessing other community-based groups.

Research findings indicated that the inclusion of peer mentors was an essential element in the development of a safe and supportive environment for participants and it is recommended that peer support be incorporated into future creative arts interventions. Our original research design was focused on outcomes, but throughout the study it became clear that the methods or processes were just as (or more) important than the outcomes. The addition of peer mentors to community-based groups may be the secret ingredient needed to build community capacity and promote inclusion for mental health consumers. It is hoped that learnings from this study can be used by others to develop future creative arts opportunities for people experiencing severe and persistent mental illness.

\section{Acknowledgements}

The research team wish to thank the Brisbane South PHN (formerly Greater Brisbane Metro South Medicare Local) for funding this research. The views and opinions expressed in this paper are those of the authors and do not necessarily reflect those of the PHN. We also acknowledge the artists, peer mentors and research staff who participated in parts of the research journey, particularly Rani Scott and Rachael Krinks. Finally, but not least, we would like to thank our research participants without whom this project would not have been possible. 


\section{Disclosure statement}

The authors declare no known/perceived conflicts of interest.

\section{References}

Appgahw 2017. Inquiry Report: Creative Health: The Arts for Health and Wellbeing. U.K.: All-Party Paliamentary Group on Arts, Health and Wellbeing.

Argyle, E. and Winship, G. 2015. Creative practice in a group setting. Mental Health and Social Inclusion, 19, 141-147.

Baldwin, C. 2005. Narrative, ethics and people with severe mental illness. Australian \& New Zealand Journal of Psychiatry, 39, 1022-1029.

Clift, S. 2012. Creative arts as a public health resource: moving from practice-based research to evidence-based practice. Perspectives in Public Health, 132, 120-127.

Elliott, J. 2005. Using narrative in social research: qualitative and quantitative approaches, SAGE, London, U.K.

Frank, A. W. 1995. The wounded storyteller: body, illness, and ethics, University of Chicago Press, Chicago, U.S.A.

Happell, B. and Bennetts, W. 2016. Triumph and adversity: Exploring the complexities of consumer storytelling in mental health nursing education. International Journal of Mental Health Nursing, 25, 546-553.

Happell, B., Bennetts, W., Platania-Phung, C. and Tohotoa, J. 2015. Consumer involvement in mental health education for health professionals: feasibility and support for the role. Journal of Clinical Nursing, 24, 3584-3593.

Kerr, D. J. R., Crowe, T. P. and Oades, L. G. 2013. The Reconstruction of Narrative Identity During Mental Health Recovery: A Complex Adaptive Systems Perspective. Psychiatric Rehabilitation Journal, 36, 108-109.

Kirkpatrick, H. 2008. A Narrative Framework for Understanding Experiences of People With Severe Mental Illnesses. Archives of Psychiatric Nursing, 22, 61-68.

Lawson, J., Reynolds, F., Bryant, W. and Wilson, L. 2014. 'It's like having a day of freedom, a day off from being ill': Exploring the experiences of people living with mental health problems who attend a community-based arts project, using interpretative phenomenological analysis. Journal of Health Psychology, 19, 765-777.

Macneil, C. and Mead, S. 2005. A Narrative Approach to Developing Standards for Trauma-Informed Peer Support. American Journal of Evaluation, 26, 231-244. 
Mcadams, D. P. 2006. The Problem of Narrative Coherence. Journal of Constructivist Psychology, $19,109-125$.

Mead, S., Hilton, D. and Curtis, L. 2001. Peer support: A theoretical perspective. Psychiatric Rehabilitation Journal, 25, 134-141.

Pernice, F. M., Biegel, D. E., Kim, J.-Y. and Conrad-Garrisi, D. 2017. The Mediating Role of Mattering to Others in Recovery and Stigma. Psychiatric Rehabilitation Journal, 40, 395-404.

Repper, J. and Perkins, R. 2009. "Recovery and social inclusion", Callaghan, P., Playle, J. and Cooper, L. (Eds.) Mental health nursing skills. Oxford University Press, Oxford, U.K.

Sayers, T. and Stickley, T. 2018. Participatory arts, recovery and social inclusion. Mental Health and Social Inclusion, 22, 149-156.

Spandler, H., Secker, J., Kent, L., Hacking, S. and Shenton, J. 2007. Catching life: the contribution of arts initiatives to recovery approaches in mental health. Journal of Psychiatric and Mental Health Nursing, 14, 791-799.

Stickley, T., Wright, N. and Slade, M. 2018. The art of recovery: outcomes from participatory arts activities for people using mental health services. Journal of Mental Health, 1-7.

Stuckey, H. L. and Nobel, J. 2010. The Connection Between Art, Healing, and Public Health: A Review of Current Literature. American Journal of Public Health, 100, 254-263.

Tarr, J., Gonzalez-Polledo, E. and Cornish, F. 2017. On liveness: using arts workshops as a research method. Qualitative Research, 18, 36-52.

Tew, J., Ramon, S., Slade, M., Bird, V., Melton, J. and Le Boutillier, C. 2011. Social Factors and Recovery from Mental Health Difficulties: A Review of the Evidence. British Journal of Social Work, 42, 443-460.

Van Lith, T. 2015. Art Making as a Mental Health Recovery Tool for Change and Coping. Art Therapy, 32, 5-12.

Van Lith, T., Fenner, P. and Schofield, M. 2011. The lived experience of art making as a companion to the mental health recovery process. Disability and Rehabilitation, 33, 652-660.

Van Lith, T., Schofield, M. J. and Fenner, P. 2013. Identifying the evidence-base for art-based practices and their potential benefit for mental health recovery: A critical review. Disability and Rehabilitation, 35, 1309-1323.

Walsh, P. E., Mcmillan, S. S., Stewart, V. and Wheeler, A. J. 2018. Understanding paid peer support in mental health. Disability \& Society, 1-19. 